\title{
Exposiciones a fluidos de riesgo en el personal de salud. Evaluación de gastos directos en su manejo
}

\author{
Teresa Vergara', Elena Véliz', Alberto Fica' y Jeannette Dabanch'
}

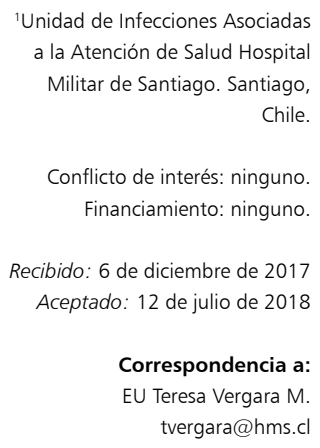

'Unidad de Infecciones Asociadas a la Atención de Salud Hospital Militar de Santiago. Santiago Chile.

Conflicto de interés: ninguno. Financiamiento: ninguno.

Recibido: 6 de diciembre de 2017 Aceptado: 12 de julio de 2018

Correspondencia a: EU Teresa Vergara M. tvergara@hms.c

\section{Exposure to risk fluids in health personnel. Evaluation of direct costs in their management}

Background: Health personnel are exposed to accidents with blood and other potentially infectious biological fluids in their clinical practice. Management of these events has high costs that are likely to be reduced. Aim: To give a general description of work accidents and their associated costs. Material and Method: All healthcare workers reports of exposure to blood or risk fluids between 2010 and 2015, were included. Health care costs were calculated. Records were analyzed in a retrospective manner. Results: 189 exposures to risk fluids were reported during the study period with an average of 31 accidents per year. $83.5 \%$ of the studied sources were negative for HIV, HBV and HCV, $12.6 \%$ was with unknown source and only $3.7 \%$ was positive for any of them. The costs associated with management of risk exposures was $\$ 2,765,890$ Chilean pesos/year $(4,274$ USD). The average cost per event was $\$ 73,171$ Chilean pesos (113 USD). Approximately $80 \%$ of this figure corresponds to basic management protocol. Conclusion: Exposure to risk fluids in health personnel is frequent and has a high cost. This cost can be reduced by the application of prevention measures to give the healthcare workers a safer environment for their daily practice.

Keywords: Costs; work accidents; exposure to risk fluids; health workers.

Palabras clave: Costos; accidentes laborales; exposiciones a fluidos de riesgo; trabajadores de la salud.

\section{Introducción}

$\mathrm{E}$ 1 personal de salud está expuesto a sangre y otros fluidos biológicos potencialmente infectantes a través de accidentes donde se expone la mucosa o piel no intacta o en forma percutánea mediante pinchazos o cortes, siendo la exposición percutánea la más frecuente de ellas ${ }^{1}$.

Estas exposiciones representan un riesgo para el personal de salud ya que existen varios agentes potenciales de contagio, incluyendo el virus de la hepatitis B (VHB), virus de la hepatitis $\mathrm{C}$ (VHC) y el virus de inmunodeficiencia humana $(\mathrm{VIH})^{1-3}$. Estos tres agentes virales son de gran importancia debido al riesgo que éstos implican en hepatitis aguda, fulminante o crónica o SIDA.

Cada uno de esto virus contiene un riesgo de seroconversión distinto ${ }^{4}$; es así como, para VIH el riesgo es aproximadamente $0,3 \%$ luego de lesiones percutáneas y menor en exposiciones sobre mucosas ${ }^{2,5}$, para VHC se describe un riesgo 1,8\%- 5\% $\%^{5,6}$. En contraste, para el VHB se ha documentado un riesgo más alto, cercano a $30 \%$ en personas no vacunadas 5 . Por ello se hace relevante la implementación de medidas preventivas como la inmunización para hepatitis $\mathrm{B}$ que permite una disminución de este riesgo a cifras cercanas a $0,3 \%{ }^{7}$.

La prevalencia en Chile para estos tres virus ha sido baja en la población general $(<1 \%)^{8}$, lo que contribuye a tener una falsa sensación de seguridad y, por ende, incumplimiento de medidas de seguridad y autocuidado por parte de los trabajadores de la salud. Sin embargo, según el último informe de ONUSIDA ${ }^{9}$, Chile ha aumentado el número anual de nuevas infecciones por el VIH en 34\%, liderando la Región de Las Américas, por encima de otros países como Guatemala (23\%), Costa Rica (16\%), Honduras (11\%) y Panamá (9\%), aumentado el riesgo de tener una exposición con una fuente positiva a este virus en los centros de salud. Además, la Organización Mundial de la Salud estima que cada año más de 3 millones de trabajadores de la salud presentan exposiciones con objetos contaminados con alguno de estos tres virus ${ }^{10}$.

Las exposiciones de este tipo no son infrecuentes en nuestro país; se describe una tasa anual de 0,9 por 100 estudiantes en un hospital universitario ${ }^{11}$ y la experiencia de un hospital que funciona como asegurador laboral ${ }^{12}$, relata haber dado atención a 615 exposiciones a fluidos de riesgo en un período de cinco meses, donde sólo 50\% de los eventos tenía fuente identificable. De éstos, $17 \%$ era positivo a alguno de los tres virus testeados. Así mismo, los afectados con fuente positiva requirieron iniciar profilaxis, la que no es inocua, existiendo mala adherencia: alrededor de 38,7\% abandonó el seguimien- 
to. Pese a ello, en ambas experiencias no se documentó seroconversión de funcionarios con posterioridad a la exposición.

Por otra parte, la atención de estos eventos implica costos importantes, tanto para el afectado como para la institución de salud y/o aseguradora de salud implicada. Las cifras varían dependiendo de la intensidad del estudio de la fuente asociada a la exposición, del resultado de la serología del paciente fuente y de la situación de vacunación para el VHB del afectado ${ }^{13-15}$. Además, existe un impacto personal, familiar, social y laboral de estos eventos, asociado al ausentismo o angustia que éstos provocan. En perspectiva, estos eventos no son inusuales; por ejemplo, en nuestra institución hasta el año 2015, las exposiciones a fluidos de riesgo biológico en el personal de salud correspondían a $30 \%$ del total de accidentes laborales reportados (datos no publicados).

A pesar de las publicaciones nacionales existentes al respecto, no existe un estudio del impacto económico directo de estas exposiciones en el personal de salud en Chile. Tal información es necesaria para dimensionar la carga de este problema en términos económicos, poner de manifiesto una vez más la necesidad de prevenir estos accidentes y dar pie a futuros estudios de costo-beneficio.

El objetivo del presente trabajo es describir aspectos generales de la accidentabilidad local y los costos directos asociados a la gestión y manejo de las exposiciones a fluidos de riesgo en el personal de salud del Hospital Militar (HOSMIL) de Santiago entre los años 2010 y 2015.

\section{Material y Método}

\section{Diseño del estudio}

Estudio descriptivo, retrospectivo. Se tomaron todas las notificaciones de exposición a fluidos de riesgo en el personal de salud entre los años 2010 y 2015 del HOSMIL de Santiago registradas en el Programa de Salud del Personal que lleva la Unidad de Prevención y Control de Infecciones Asociadas a la Atención de Salud (IAAS) de la instalación. Se excluyeron alumnos y funcionarios de otras instalaciones de salud del Ejército.

Se definió como exposición a fluido de riesgo, una punción o corte con un elemento corto punzante contaminado con fluido de riesgo (sangre principalmente, o líquido de cavidad estéril o cualquier fluido que contenga sangre) y las salpicaduras sobre piel no indemne o mucosas con estos fluidos. Se excluyó otros tipos de fluidos.

\section{Manejo de eventos}

Las exposiciones se manejaron de acuerdo al protocolo local que separa los eventos según tipo de fuente: conocida o desconocida. El protocolo básico de atención considera el estudio de la fuente en forma simultánea para VHB, VHC y VIH mediante serología con la atención médica de estos eventos la que se realiza en forma permanente en el hospital. Los eventos excluyentes se clasificaron en tres grupos de acuerdo a su manejo.

\section{Exposición con fuente negativa}

Corresponde a una exposición donde se conoce el origen del fluido y la serología del paciente fuente $\mathrm{u}$ origen resulta finalmente ser negativa para los tres virus estudiados. Esta situación no constituye riesgo para el funcionario y permite cerrar el caso en la primera consulta, sin requerir seguimiento.

\section{Exposición con fuente positiva}

Corresponde a una exposición donde se conoce el origen del fluido y éste es positivo para uno o más virus estudiados. Esta situación es de riesgo ya que existe la posibilidad de seroconversión del afectado. En el caso de VHB, el riesgo tiene directa relación con el estado de inmunización del afectado para este virus. En caso de ser positivo para VIH se requiere de indicación de profilaxis antiviral y seguimiento. El detalle del protocolo se presenta en las Figuras 1 a 3.

\section{Exposición con fuente desconocida}

Corresponde a una exposición donde no se conoce el origen del fluido de riesgo. Localmente esta situación es considerada de bajo riesgo dada la baja prevalencia de portadores crónicos de VHB o de personas con infección por VHC o VIH en Chile, similar a la población cautiva que el hospital atiende (hospital general con mayoría

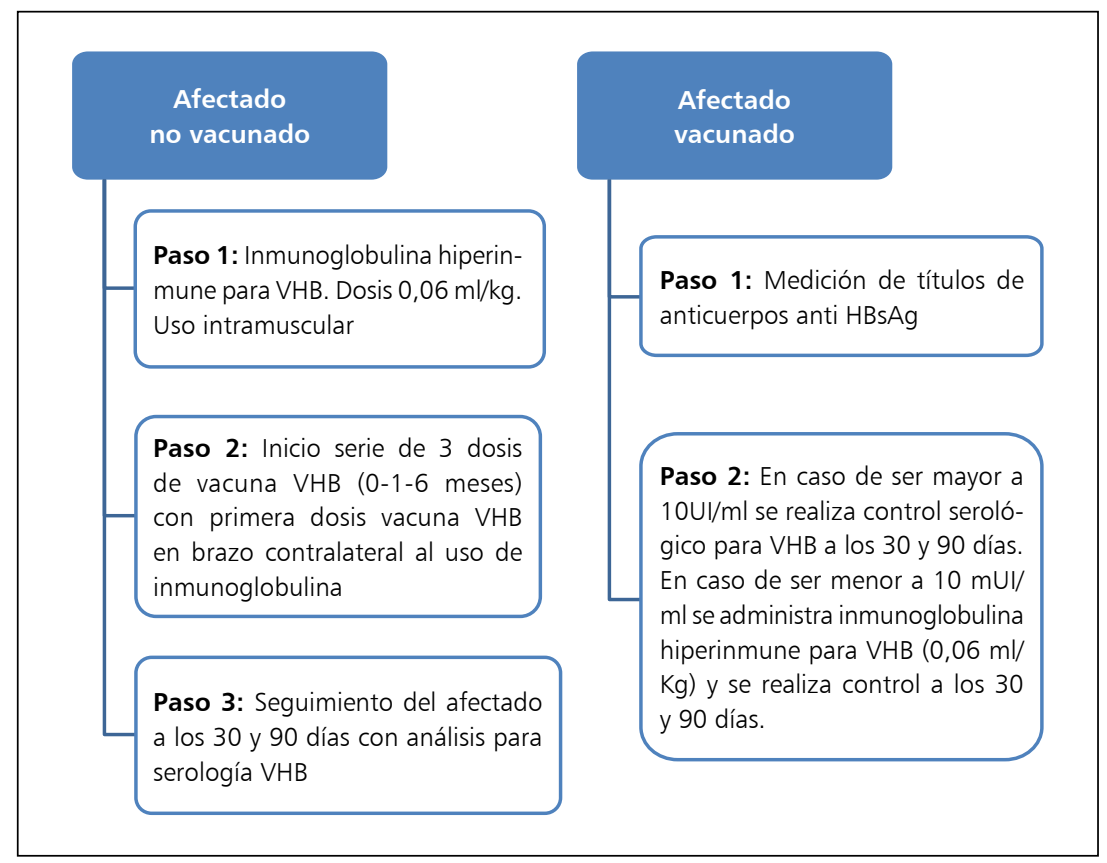

Figura 1. Protocolo de manejo, exposición con fuente positiva a VHB. 


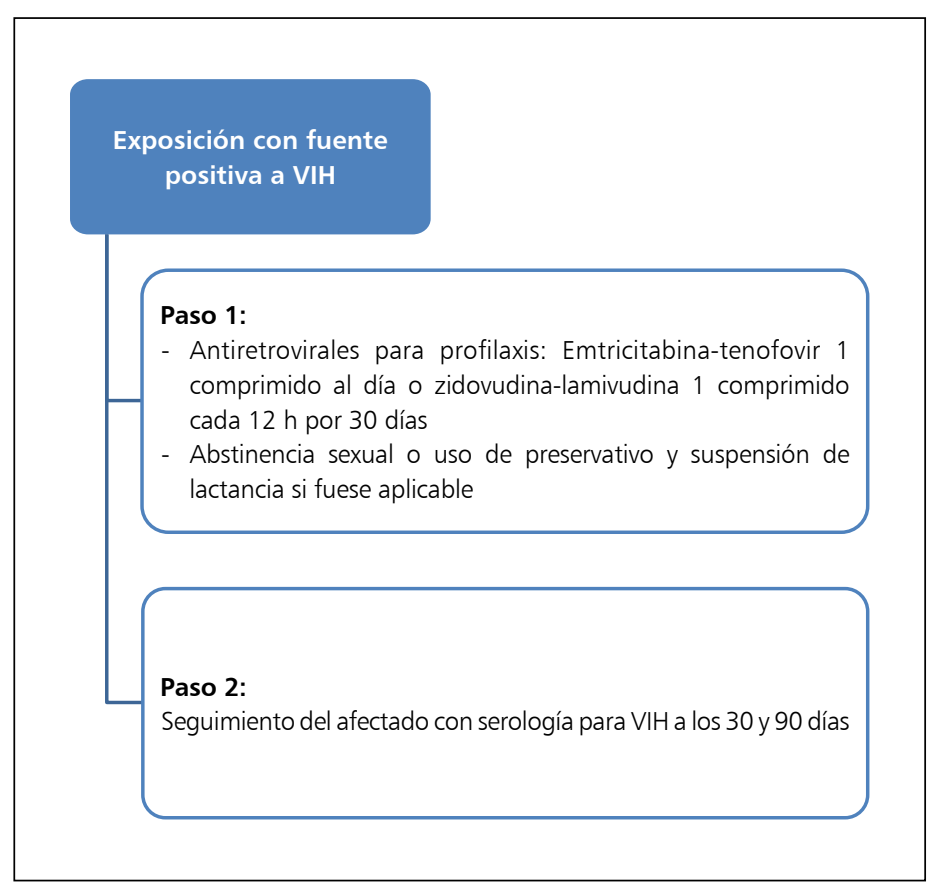

Figura 2. Protocolo de manejo, exposición fuente positiva VIH.

población adulta). Estos eventos son evaluados caso a caso para determinar seguimiento o alta. En aquellos no vacunados, se indica la inmunización para $\mathrm{VHB}^{16-18}$ (Figura 4).

\section{Evaluación de gastos directos}

Se analizaron los costos asociados a estos eventos los que contemplan: atención médica inicial y de seguimiento, exámenes de laboratorio del paciente fuente y del funcionario en caso de que la fuente sea positiva a alguno de los virus estudiados. Los valores unitarios año 2016 se presentan en la Tabla 1.

\begin{tabular}{ll}
$\begin{array}{l}\text { Tabla 1. Costos directos por concepto de atención básica de exposicio- } \\
\text { nes a fluidos de riesgo }\end{array}$ & Costo \\
\hline Ítem & $\$ 24.980$ \\
\hline Atención del afectado en servicio de urgencias & \\
\hline $\begin{array}{l}\text { Estudio serológico de la fuente (3 virus) } \\
\text { VIH }\end{array}$ & $\$ 6670$ \\
VHB & $\$ 6540$ \\
VHC & $\$ 10.050$ \\
Toma de muestra & $\$ 1.020$ \\
\hline Consulta y consejería infectológica & $\$ 19.660$ \\
\hline Serología del afectado en caso de fuente positiva & $\$ 24.250$ \\
\hline Seguimiento del afectado si se requiere (valor por consulta) & $\$ 19.660$
\end{tabular}

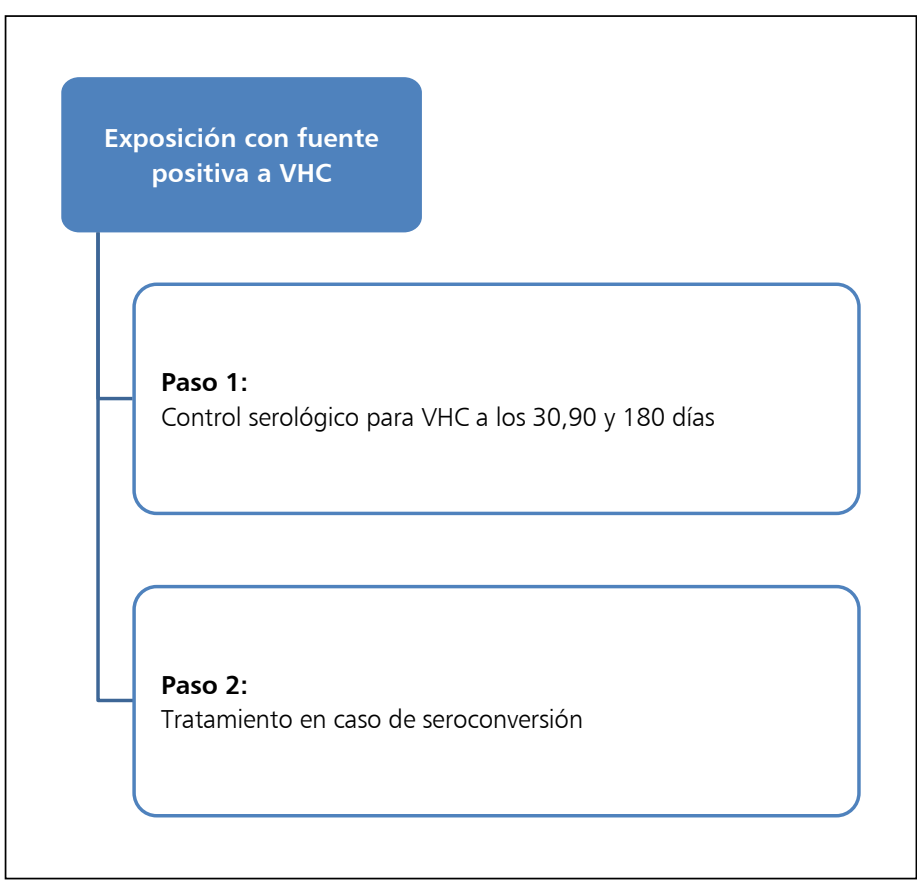

Figura 3. Protocolo de manejo, exposición fuente positiva VHC.

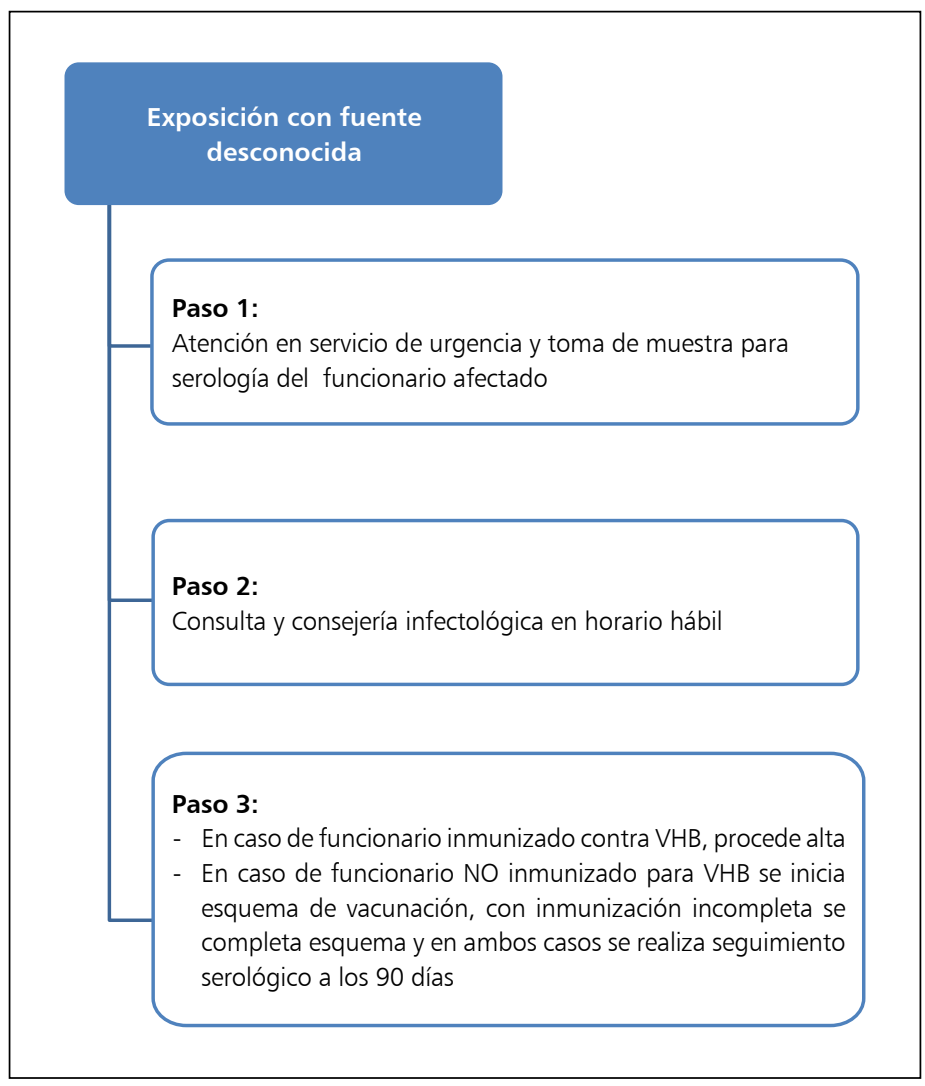

Figura 4. Protocolo de manejo, exposición fuente desconocida. 


\section{Resultados}

\section{Generalidades y distribución}

Se reportaron 189 exposiciones a fluido de riesgo durante el período estudiado con un promedio de 31 accidentes anuales (mediana 32, rango 20 a 38). La incidencia de eventos fue de 3 por 100 expuestos. El estamento mayormente afectado fue el de enfermería con una tasa de incidencia de 5,4 por 100 expuestos, luego el estamento médico (tasa de incidencia de 2,7 ) y posteriormente los técnicos paramédicos (tasa de incidencia 2,6).

En relación a la serología de la fuente, $83,5 \%$ fue negativa para los tres virus analizados y sólo $3,7 \%$ fue positiva a alguno de ellos (siete casos). Así, cuatro de los siete casos correspondieron a VHC, dos a VHB y uno a VIH. No hubo seroconversión en los funcionarios afectados, completando todos ellos el seguimiento por protocolo.

En relación a las exposiciones con fuente desconocida, en el período se reportó un total de 24 eventos (12,6\%). Aquellos que tenían su vacunación completa para el VHB $(n=18)$ se manejaron con atención de urgencia para evaluar la lesión y consejería. Los que no tenían vacunación o la tenían incompleta $(\mathrm{n}=6)$ se manejaron completando su esquema de vacunación y con un control serológico a los 90 días (para los tres virus). Cabe destacar que en aquellos accidentes donde no se pudo testear la fuente por diversas causas, los expuestos fueron tratados como fuente desconocida.

\section{Cumplimiento de medidas de auto-cuidado}

Vacunación contra hepatitis B: Al momento del accidente, $87,4 \%$ del personal afectado se encontraba con su esquema de vacunación completo para el VHB, $10,5 \%$ no estaba vacunado y sólo $1,6 \%$ tenía un esquema incompleto, que fue necesario regularizar con posterioridad al accidente.

Uso de elementos de protección personal (EPP): Este dato se obtuvo desde el año 2011 en adelante, reportando que $54,3 \%$ del personal afectado utilizaba barreras al momento del accidente y $45,7 \%$ no las utilizaba. La tendencia en el cumplimiento del uso de EPP es más bien errática, dado que el año 2011 existía $40 \%$ de cumplimiento y aumentó sobre $60 \%$ en los años 2012 y 2013; sin embargo, en el 2014 y 2015 el cumplimiento se redujo a 50\%.

\section{Costos directos}

El costo total en el período de estudio por concepto de manejo de exposiciones de riesgo fue de $\$ 13.829 .450$ (Tabla 2) lo que da un costo anual del programa de aproximadamente $\$ 2.765 .890$ pesos chilenos $(4,274$ USD) y de $\$ 73.171$ aproximadamente por evento manejado (113 USD). Cerca de $80 \%$ de esta cifra corresponde al protocolo de manejo de funcionarios afectados con fuente negativa $(\mathrm{n}=158), 12,6 \%$ al manejo de afectados con fuente desconocida ( $n=24)$ y $3,7 \%$ al manejo de afectados con fuente positiva $(n=7)$ (Tabla 2). Cabe destacar que la atención de mayor costo que se realizó en nuestra institución fue por una exposición a fuente positiva por VIH.

En el caso de los 24 expuestos a fuente desconocida, los 18 que se encontraban vacunados, se les dio de alta después de la consejería infectológica. De los seis funcionarios que no se encontraban inmunizados, cinco iniciaron esquema de vacunación y un funcionario se negó a la vacunación. De los seis no inmunizados, cinco tuvieron seguimiento a los 90 días y un funcionario no tuvo seguimiento dado que renunció a la institución antes de completar los 90 días post exposición (Tabla 2).

\section{Discusión}

Nuestras cifras de accidentabilidad durante el período estudiado son similares a publicaciones nacionales existentes, en relación a la frecuencia promedio en número absoluto, dado que instituciones similares reportan entre 31 y 37 exposiciones anuales $^{5,11}$ con excepción de una

\begin{tabular}{|c|c|c|c|c|}
\hline Fuente & $\mathbf{n}$ & Especificaciones & Costo por caso & Costo total \\
\hline Negativa & 158 & & $\$ 68.890$ & $\$ 10.884 .620$ \\
\hline \multirow[t]{2}{*}{ Desconocida } & 24 & 18 vacunados contra Hepatitis B & $\$ 68.890$ & $\$ 1.240 .020$ \\
\hline & & $\begin{array}{l}6 \text { NO vacunados de los cuales: } \\
5 \text { inician esquema y terminan el seguimiento } \\
1 \text { no se vacuna y no completa seguimiento }\end{array}$ & $\begin{array}{l}\$ 118.550 \\
\$ 68.890\end{array}$ & $\begin{array}{l}\$ 661.640 \\
\$ 68.890\end{array}$ \\
\hline Positiva & 7 & $\begin{array}{l}4 \mathrm{VHC} \\
2 \mathrm{VHB} \text { (vacunados) } \\
1 \mathrm{VIH} \text { (tratado) }\end{array}$ & $\begin{array}{l}\$ 92.190 \\
\$ 97.440 \\
\$ 479.530\end{array}$ & $\begin{array}{l}\$ 368.760 \\
\$ 194.880 \\
\$ 479.530\end{array}$ \\
\hline Total eventos & 189 & & Total costos & $\$ 13.829 .450$ \\
\hline
\end{tabular}


experiencia pediátrica que reporta alrededor de 16 exposiciones al año ${ }^{19}$. Sobre publicaciones internacionales, una experiencia mexicana refirió un promedio de 43 accidentes anuales ${ }^{20}$.

Universalmente, la fuente involucrada en estos accidentes se asocia en $39 \%$ a pacientes con infección por VHC, $37 \%$ a pacientes con infección por VHB, y 4,4\% a pacientes con infección por $\mathrm{VIH}^{21}$. Para la región de las Américas se considera que 61.000, 57.000 y 23.000 eventos ocurren con una fuente positiva para HBV, VHC, y VIH, respectivamente, cifras no alentadoras debido a la angustia que generan los costos asociados a su manejo y el riesgo potencial de transmisión ${ }^{21,22}$. Sin embargo, nuestra realidad local es bastante distinta ya que sólo 3,7\% de los accidentes estuvo expuesta a fuente positiva con un total de siete accidentes, donde ninguno seroconvirtió durante el seguimiento.

Los costos involucrados en el manejo de la exposición a fluido de riesgo biológico están relacionados con las medidas instauradas para el manejo de los funcionarios expuestos, de acuerdo a los protocolos de cada institución, lo que deben estar basados en la mejor evidencia disponible. También se debe hacer referencia al tipo de agentes prevalentes y el tipo de fuente involucrada en el accidente y con el número de eventos que se enfrenta anualmente. Aproximadamente $80 \%$ de los gastos se asocian al manejo inicial de la exposición y a las exposiciones con fuente positiva ya que son las que involucran mayor gasto en el manejo.

Es así como a nivel local los costos pueden ir desde la atención básica de un afectado con fuente negativa o fuente desconocida con un afectado con esquema de inmunización completa \$ 68.890 (108 USD), hasta la atención y tratamiento de un afectado con fuente positiva para VIH que puede costar entre \$ 168.512 (266 UDS) y $\$ 479.530$ (757 USD) dependiendo del medicamento que se tenga a disposición.

Los costos reportados en nuestro trabajo son superiores a los documentados nacionalmente ${ }^{11}$; sin embargo, las cifras están muy por debajo de lo que reportan instituciones europeas donde el manejo de exposiciones con fuente negativa duplica nuestras cifras y en caso de fuente positiva con VIH puede llegar a triplicarlas ${ }^{13}$.

En el caso de Corea, como un ejemplo de lo que ocurre en el territorio asiático, los costos por evento también son superiores a los nuestros y el programa de atención genera un costo anual de 884.385 UDS, lo cual constituye una gran carga para ellos que ha llevado a intensificar las medidas preventivas ${ }^{15}$.

Una realidad europea (Belga) estima los costos por evento en un rango de 320 USD a 2.319 USD dependiendo de la serología del paciente fuente ${ }^{22}$ cuya suma también supera nuestra realidad.

En Atlanta (E.U.A.), en el año 2007, O’Malley realizó una estimación de costo tomando como universo cuatro centros hospitalarios con un total de 3.500 camas. En dicha oportunidad se estimó que el costo por evento fue de 376 USD con un rango de 71 USD a 4.838 USD, dependiendo de la serología del paciente fuente. El costo mayor calculado en este trabajo es seis veces superior a nuestro esquema de tratamiento más caro (correspondiente a fuente positiva para VIH de alto riesgo, con el tratamiento más costoso 741 USD). Sin embargo, se describe haber manejado trabajadores expuestos con fuente VIH (+) con co-infecciones a VHB y VHC lo que aumenta considerablemente la complejidad del tratamiento y el costo asociado ${ }^{14}$

Un análisis español de costo menciona que los gastos por concepto de la gestión de este tipo de accidentes son de 457 USD con un rango entre 202 y 1.772 USD dependiendo de la fuente, cuyas cifras son superiores a las nuestras. En el caso de fuente positiva a VHB con afectado inmunizado, el costo medio fue de 550 USD y en el caso de un afectado sin inmunización un costo medio de 785 USD siendo la profilaxis post exposición la que constituyó el mayor porcentaje de los costos, concluyendo además que es de gran relevancia una evaluación de riesgo adecuada a los afectados para evitar seguimientos innecesarios ${ }^{13}$.

Cabe destacar que las diferencias notables en costo entre los reportes extranjeros y nuestra experiencia están dadas primordialmente por el gran número de fuentes positiva a virus de transmisión sanguínea involucradas en estos accidentes, principalmente $\mathrm{VIH}$ y co-infecciones con VHB y/o $\mathrm{VHC}^{14}$, al manejo de exposiciones con fuente desconocida como si fueran positivas administrando profilaxis y al gran número de afectados con esquema de vacunación incompleto para $\mathrm{VHB}^{13}$, situaciones que son menos frecuentes en nuestra realidad.

Una revisión sistemática efectuada en Italia, publicada en el año 2016, muestra costos significativos, tanto directos como indirectos, asociadas al manejo de las lesiones por aguja, 425 USD sólo por costo directo (rango 48 USD a 1.516 USD) y 747 USD por caso al considerar los costos agregados, por lo cual, los esfuerzos deben focalizarse en la prevención de estos eventos. También hace alusión a la incorporación de dispositivos de ingeniería de seguridad, cuyo gasto pudiese compensarse con los ahorros de una menor accidentabilidad ${ }^{13,23}$. Estas cifras son superiores a las calculadas en nuestro estudio y cabe destacar que localmente no se ha evaluado el costo-beneficio del uso de dispositivos de seguridad.

Al comparar el costo medio por evento manejado en nuestra institución con el resto de las publicaciones internacionales, nuestra cifra sigue siendo inferior a las reportadas, siendo sólo la publicación chilena la que arroja costos inferiores, tal como se muestra en la Tabla 3.

Un estudio italiano muestra la rentabilidad del uso de 
dispositivos de seguridad para la prevención de punciones por aguja en el personal de salud, mostrando un descenso que va desde 63 a 100\%, obteniendo como resultado una gran ventaja económica y en términos de prevención de accidentes $^{24}$. Sin embargo, una revisión sistemática efectuada en E.U.A. en el año 2014, concluyó que la evidencia existente en relación al uso de dispositivos de seguridad para prevención de accidentes es de baja calidad. Esto no quiere decir que estos dispositivos sean ineficaces, sino que es necesario disponer de estudios de mejor calidad que los avalen y evaluar rentabilidad-eficacia localmente en cada institución ${ }^{25}$.

Ya están disponibles algunos reportes internacionales de los beneficios económicos que podría tener la implementación de estos dispositivos ya que en una experiencia belga se redujo la accidentabilidad y los ahorros por este concepto compensaron con creces los mayores costos de adquisición de los dispositivos de seguridad, produciendo un ahorro global estimado a cinco años de $€ 51,710$. (55.146 USD, \$35.679.900)26. A su vez, Cooke y Stephens en E.U.A. lograron reportar una reducción de la accidentabilidad de 43,4 a 100\% al cambiar las agujas hipodérmicas convencionales por unas de seguridad que cubrieran la punta después del uso, lo que mostró ahorros netos, impacto presupuestario favorable y rentabilidad general $^{27}$.

Otros autores destacan, además, los beneficios que estos dispositivos pueden traer en el ámbito laboral de los funcionarios ya que disminuyen el temor del operador a sufrir un accidente, disminuyendo así la inseguridad y otros costos intangibles ${ }^{28}$.

Nuestra realidad local muestra que $54 \%$ de los eventos fueron producidos por una aguja con lumen; tal vez sería interesante frente a nuestra problemática probar algún sistema que permitiera encapsular automáticamente estas agujas con posterioridad a su uso, tomado en cuenta que la mayoría de estos accidentes se produce al recapsular manualmente este elemento o por no eliminarlo en forma inmediata en el reservorio correspondiente.

Por otra parte, $18 \%$ de los eventos fue con una aguja sin lumen (de sutura), en este caso se podría optimizar el uso de los "contadores de agujas" (dispositivos cuyo objetivo es llevar la contabilidad de las agujas utilizadas en la mesa y prevenir accidentes cortopunzantes por el extravío de una de ellas), en el campo quirúrgico, en conjunto con potenciar el traspaso de material cortopunzante sobre superficies entre operadores. De esta manera se podría abordar 72\% (n: 136 de 189) de los accidentes producidos por agujas y a su vez evaluar utilidad e impacto de la incorporación de estas tecnologías.

Algunas de las limitaciones que presenta este estudio corresponden al impacto de los costos intangibles tales como ausentismo laboral del funcionario ocasionado por el traslado o por licencia médica, los que no fueron
Tabla 3. Costos por evento de los distintos trabajos consultados

\begin{tabular}{cccc}
\hline Referencia & Año & Costo medio por evento en USD \\
17 & 2010 & 1,58 USD \\
11 & 2005 & 457,58 USD \\
13 & 2013 & 237 & USD \\
21 & 2014 & 320,78 USD \\
12 & 2007 & 376 & USD \\
23 & 2016 & 425 & USD \\
\hline
\end{tabular}

calculados, al igual que los costos indirectos tales como la pérdida de la productividad y el impacto cualitativo en la calidad de vida del afectado manifestado por la angustia, ansiedad, irritabilidad, en el período de seguimiento, la afección a las respectivas familias y vida de pareja ${ }^{5}$, lo que sin duda tiene un gran valor.

En conclusión, las exposiciones a fluidos de riesgo en el personal de salud son frecuentes, y la atención de los afectados tiene un costo importante. En nuestra institución, el costo medio por evento es de $\$ 73.171$ y $80 \%$ de los costos del programa está constituido por el manejo inicial de la exposición. El otro ítem que determina altos costos es el manejo de los afectados con fuente positiva, lo que en nuestra experiencia alcanza a 3,7\% del total de los costos. Estas cifras son susceptibles de reducir en la medida que se previenen las exposiciones a fluidos a través de las distintas estrategias validadas ${ }^{29-31}$ $\mathrm{y}$ al otorgar al trabajador de la salud un escenario seguro para su práctica diaria.

\section{Resumen}

Introducción: El personal de salud está expuesto a experimentar accidentes con sangre y otros fluidos biológicos potencialmente infectantes en su práctica clínica; la atención de estos eventos implica costos importantes, los que son susceptibles de reducir. $O b$ jetivo: Describir aspectos generales de los accidentes laborales y los costos asociados al manejo. Material $y$ Método: Estudio descriptivo, retrospectivo. Se tomaron las notificaciones de exposición a fluidos en el personal de salud entre los años 2010 y 2015 y se calcularon los costos por concepto de atención de los afectados. Resultados: Se reportaron 189 exposiciones a fluido de riesgo durante el período estudiado con un promedio de 31 accidentes anuales. El 83,5\% de las fuentes estudiadas fueron negativas para VIH, VHB y VHC, 12,6\% fue accidente con fuente desconocida y sólo $3,7 \%$ fue positivo a alguno de estos virus. Los costos asociados al manejo de las exposiciones de riesgo 
fueron de $\$ 2.765 .890$ anuales (4,274 USD) y un costo medio de $\$ 73.171$ por evento manejado (113 USD); cerca de $80 \%$ de esta cifra corresponde al protocolo de manejo básico. Conclusión: Las exposiciones a fluidos de riesgo en el personal de salud son frecuentes, y la atención de los afectados tiene un costo importante. Estas cifras son susceptibles de reducir en la medida que se previenen las exposiciones a fluidos, a través de las distintas estrategias validadas y al otorgar al trabajador de la salud un escenario seguro para su práctica diaria.

\section{Referencias bibliográficas}

1.- Coppola N, De Pascalis S, Onorato L, Calò F, Sagnelli C, et al. Hepatitis B virus and hepatitis $\mathrm{C}$ virus infection in healthcare workers. World J Hepatol. 2016; 8: 273-81. doi: 10.4254/wjh. v8.i5.273.

2.- Henderson D K, Fahey B J, Willy M, Schmitt J M, Carey K, et al. Risk for occupational transmission of human immunodeficiency virus type 1 (HIV-1) associated with clinical exposures. A prospective evaluation. Ann Intern Med 1990; 113: 740-6.

3.- Sulkowski M S, Ray S C, Thomas D L. Needlestick transmission of hepatitis C. JAMA 2002; 287: 2406-13.

4.- Morales E. Accidentes laborales con exposición a fluidos corporales: Características y circunstancias de su ocurrencia en el personal de salud del Hospital Clínico Regional de Valdivia, durante el año 2002. Escuela de Enfermería, Facultad de Medicina de la Universidad Austral. 2004. http://cybertesis. uach.cl/tesis/uach/2004/fmm828a/pdf/ fmm828a.pdf (accedido el 16 septiembre de 2016).

5.- Villarroel J, Bustamante M C, Manríquez I, Bertoglia M P, Mora M, et al. Exposición laboral a fluidos corporales de riesgo en el Hospital Clínico Félix Bulnes Cerda durante 11 años de estudio. Rev Chilena Infectol 2012; 29: 255-62. http://dx.doi.org/10.4067/S071610182012000300002.

6.- Medeiros W P, Setúbal S, Pinheiro P Y, Dalston M O, Bazin A R. Occupational hepatitis C seroconversions in a Brazilian hospital. Occup Med (Lond) 2012; 62: 655-7. doi: 10.1093/ occmed/kqs 105 .

7.- Chen W, Gluud C. Vaccines for preventing hepatitis B in health-care workers. Cochrane Database Syst Rev 2005 Oct 19:CD000100.

8.- Monge M, Rodríguez E. Guía preventiva de recomendaciones para trabajadores(as) sanitarios en manejo de material Cortopunzante. Departamento Salud Ocupacional M. Alfaro Sección Riesgos Biológicos, Chile. http://www. ispch.cl/sites/default/files/u5/Guia_Preventiva_ Cortopunzantes.pdf (último acceso 1 de febrero de 2017).

9.- Joint United Nations Programme on HIV/AIDS (UNAIDS). Ending AIDS Progress towards the 90-90-90 targets. GLOBAL AIDS UPDATE, 2017. http://www.unaids.org/ sites/default/
files/media_asset/Global_AIDS_update_2017_ en.pdf.

10.- Montella E, Schiavone D, Apicella L, Di Silverio P, Gaudiosi M, et al. Cost-benefit evaluation of a preventive intervention on the biological risk in health: the accidental puncture during the administration of insulin in the University Hospital "Federico II" of Naples. Ann Ig 2014; 26: 272-8. doi: 10.7416/ ai.2014.1985

11.- Fica C A, Jemenao P M I, Ruiz R G, Larrondo $\mathrm{L}$ M, Hurtado H C, et al. Accidentes de riesgo biológico entre estudiantes de carreras de la salud. Cinco años de experiencia. Rev Chilena Infectol. 2010; 27: 34-9. http://dx.doi. org/10.4067/S0716-10182010000100005

12.- Cerón I. Seguimiento de accidentes laborales con exposición a fluidos corporales con riesgo biológico manejados en el hospital del trabajador de Santiago. Resultados preliminares. En J. Dabanch (Presidencia), XXXI Congreso Chileno de Infectologia. Noviembre 2014. Puerto Varas, Chile. Abstract. CO-4, pág 16. http://www.sochinf.cl/portal/ templates/sochinf2008/documentos/2014/ Libro_congreso_2014.pdf

13.- Solano V M, Hernández M J, Montes F J, Arribas J L. Actualización del coste de las inoculaciones accidentales en el personal sanitario hospitalario. Gac Sanit 2005; 19: 2935. http://scielo.isciii.es/pdf/gs/v19n1/original4. pdf

14.- O’Malley E M, Scott R D 2nd, Gayle J, Dekutoski J, Foltzer M, et al. Costs of management of occupational exposures to blood and body fluids. Infect Control Hosp Epidemiol 2007; 28: 774-82. DOI: $10.1086 / 518729$

15.- Oh H S, Yoon Chang S W, Choi J S, Park E S, Jin H Y. Costs of postexposure management of occupational sharps injuries in health care workers in the Republic of Korea. Am J Infect Control 2013; 41: 61-5. doi: 10.1016/j. ajic.2012.01.030.

16.- Muñoz G, Velasco M, Thiers V, Hurtado C, Brahm J, Larrondo-Lillo M, et al. Prevalencia y genotipos del virus $\mathrm{C}$ de la hepatitis en donantes de sangre y en pacientes con enfermedad hepática crónica y hepatocarcinoma en población chilena. Rev Med Chile 1998; 126: 1035-42.

17.- Zunino E. Epidemiología de la hepatitis $B$ en Chile y esquemas de vacunación en
Latinoamérica. Rev Chilena Infect 2002; 19 : 140-55. http://dx.doi.org/10.4067/S071610182002000300002.

18.- Ibarra H. Cambios en la epidemiología de las hepatitis virales en Chile y consideraciones en estrategias de prevención. Rev Med Chile 2007; 135: 229-39. http://dx.doi.org/10.4067/S003498872007000200013.

19.- Mendoza C, Barrientos C, Vásquez R, Panizza V. Exposición laboral a sangre y fluidos corporales. Experiencia en un hospital pediátrico. Rev Chilena Infect 2001; 18: 28-34. http://dx.doi.org/10.4067/S071610182001000100004.

20.- Huertas M, Rivera-Morales I, Romero C, Ponce-de-León S. Accidentes ocupacionales e incidencia de infección por VIH y hepatitis B y $\mathrm{C}$ en una institución mexicana. Rev Invest Clin 1995; 47: 181-7.

21.- Prüss-Ustün A, Rapiti E, Hutin Y. Estimation of the global burden of disease attributable to contaminated sharps injuries among health-care workers. Am J Ind Med 2005; 48: 482-90. DOI: 10.1002/ajim.20230.

22.- Elseviers M, Arias-Guillén M, Gorke A, Arens H J. Sharps injuries amongst healthcare workers: review of incidence, transmissions and costs. J Ren Care 2014; 40: 150-6. doi: 10.1111/ jorc. 12050.

23.- Mannocci A, De Carli G, Di Bari V, Saulle R, Unim B, et al. How Much do needlestick injuries cost? A systematic review of the economic evaluations of needlestick and sharps injuries among healthcare personnel. Infect Control Hosp Epidemiol 2016; 37: 635-46. doi: 10.1017/ice.2016.48.

24.- Montella E, Schiavone D, Apicella L, Di Silverio P, Gaudiosi M, et al. Cost-benefit evaluation of a preventive intervention on the biological risk in health: the accidental puncture during the administration of insulin in the University Hospital "Federico II" of Naples. Ann Ig. 2014; 26: 272-8.

25.- Lavoie M, Verbeek J, Pahwa M. Devices for preventing percutaneous exposure injuries caused by needles in healthcare personnel. Cochrane Database Syst Rev. 2014; 3: CD009740. doi: 10.1002/14651858.CD009740. pub2

26.- Hanmore E, Maclaine G, Garin F, Alonso A, Leroy N. Economic benefits of safetyengineered sharp devices in Belgium - a budget impact model. BMC Health Serv Res. 2013 
Nov 25; 13: 489. doi: 10.1186/1472-6963-13489.

27.- Cooke C E, Stephens J M. Clinical, economic, and humanistic burden of needlestick injuries in healthcare workers. Med Devices (Auckl) 2017; 10: 225-35. doi: 10.2147/MDER.

28.- Glenngård A H, Persson U. Costs associated with sharps injuries in the Swedish health care setting and potential cost savings from needle-stick prevention devices with needle and syringe. Scand J Infect Dis 2009; 41: 296-302. doi: 10.1080/00365540902780232.

29.- Sannoh S, Clones B, Muñoz J, Montecalvo M, Parvez B. A multimodal approach to central venous catheter hub care can decrease catheter-related bloodstream infection. Am J Infect Control 2010; 38: 424-9. doi: 10.1016/j. ajic.2009.07.014.

30.- Sjöberg M, Eriksson M. Hand disinfectant practice: the impact of an education intervention. Open Nurs J 2010; 4: 20-4. doi: 10.2174/1874434601004010020.

31.- Lobo R, Levin A, Oliveira M, Gomes L, Gobara S, Park M, et al. Evaluation of interventions to reduce catheter-associated bloodstream infection: continuous tailored education versus one basic lecture. Am J Infect Control 2010; 38: 440-8. doi: 10.1016/j. ajic.2009.09.013. 